\title{
THE SCHUR INDEX OF THE $p$-REGULAR CHARACTERS OF THE BOREL SUBGROUP
}

\section{G. I. LEHRER}

\begin{abstract}
Let $B$ be the group of $\mathbf{F}_{q}$-rational points of the Borel subgroup of a connected reductive group defined over the finite field $F_{q}$. It is shown that under appropriate conditions, all irreducible characters of $B$ which have degree prime to $q$ have Schur index one.
\end{abstract}

Let $\mathbf{G}$ be a connected reductive linear algebraic group defined over the finite field $F_{q}$, with corresponding Frobenius map $F$. We assume that $\mathbf{G}$ has connected centre $\mathbf{Z}$ and that the characteristic $p$ is good for $\mathbf{G}$. If $\mathbf{H}$ is a closed subgroup of $\mathbf{G}, H$ will denote its group of $\mathbf{F}_{q}$-rational points; for a finite group $H$, a complex irreducible character is $p$-regular if $p$ does not divide its degree; $m_{p}(H)$ is the number of such characters. It was shown in [2] that if B is an $F$-stable Borel subgroup of $\mathbf{G}$, then $m_{p}(B)=m_{p}(G)$, verifying a special case of Alperin's conjecture for finite groups. Ohmori [3] has shown that the $|Z| q^{l}$ (where $l$ is the semisimple rank of $\mathbf{G}$ ) $p$-regular characters of $G$ all have Schur index one, i.e. can be realized in their field of characters. In this note we prove the corresponding result for $p$-regular characters of $B$, viz:

THEOREM. The p-regular characters of $B$ all have Schur index one.

In the proof, we use the notation of [1] and [2]. $B$ is the semidirect product $\mathbf{T} \ltimes \mathbf{U}$, where $\mathbf{U}$ is a maximal unipotent subgroup of $\mathbf{G}$. Correspondingly, $B=T \ltimes U$, where $U$ is a $p$-group and $T$ an abelian $p^{\prime}$-group.

Proposition 1. Let $\chi$ be a p-regular character of $B$. Then $\chi=(\mu \phi)^{B}$, where $\mu$ is a linear character of $U$ and $\phi$ is a character of the centralizer $T(\mu)$ of $\mu$ in $T$. (Here $\mu \phi$ is a character of $T(\mu) \ltimes U)$.

This is elementary and was noted in [2].

Corollary $1^{\prime}$. (i) $\left.\chi\right|_{T(\mu) \cdot U}=\varphi \cdot \Sigma_{t \in T / T(\mu)} \mu^{t}$;

(ii) $\chi$ vanishes outside $T(\mu) \cdot U$.

Proof. (i) is a simple application of Frobenius' formula for induced characters, and (ii) follows since $T(\mu) \cdot U$ is normal in $B$.

Proposition 2. $\Sigma_{t \in T / T(\mu)} \mu^{t}$ takes rational values on $U$.

Received by the editors January 31, 1978.

AMS (MOS) subject classifications (1970). Primary 20G40, 20G05; Secondary 22E45.

(C) 1979 American Mathematical Society 0002-9939/79/0000-0401/\$01.75 
Proof. We have a canonical isomorphism [1, p. 258] $\eta: U / U^{\prime} \rightarrow X_{1}$ $\times \cdots \times X_{s}$, where $X_{i} \simeq\left(F_{q_{i}}\right)^{+}$. Thus we speak of the support of $\mu$, defined as $\left\{i \mid \mu\left(X_{i}\right) \neq 1\right\}$. Because of our assumption of connected centre, $T$ acts transitively on the set of linear characters of $U$ with given support (by the argument used to prove Theorem $B^{\prime}$ in [1]). Hence $\Sigma_{t \in T / T(\mu)} \mu^{t}$ is the sum of all linear characters of $U$ with fixed support $I$. By applying Galois automorphisms, one sees that this sum always takes rational values.

For any character $\xi$ of some finite group, denote by $\mathbf{Q}(\xi)$ the algebraic extension of $\mathbf{Q}$ obtained by adjoining all the values of $\xi$. This is the character field of $\xi$. From Proposition 2 we have immediately:

CoROllary $2^{\prime}$. If $\chi$ is a p-regular character of $B$, and $\phi$ is as in Proposition 1 , then $\mathbf{Q}(\chi)=\mathbf{Q}(\phi)$.

LEMMA 3. The restriction of $\chi$ to $T$ is $\phi_{T(\mu)}^{T}$.

Proof. This follows directly from Corollary $1^{\prime}$ by evaluation of $\left.\chi\right|_{T}$, or by applying Mackey's subgroup theorem.

LEMMA 4. $\phi_{T(\mu)}$ has an extension $\bar{\phi}$ to $T$, such that $\mathbf{Q}(\bar{\phi})=\mathbf{Q}(\phi)$.

Proof. If $Z$ is trivial then $T=T_{1} \times T(\mu)$, since $T$ is a direct product of the groups $\mathrm{F}_{q^{n_{i}}}^{*}$ and the condition $t \in T(\mu)$ is that certain components be trivial. In the general case, since the $\mathbb{Z}$-span of the fundamental roots has a Frobenius-invariant complement in the character group $X(T)$, we have $T \simeq Z$ $\times T / Z$; using Lang's theorem it follows that $T \simeq Z \times T / Z$. Hence $T(\mu)$ is again a direct factor (containing $Z$ ) and if $T=T(\mu) \times T_{1}$, we may take $\bar{\varphi}\left(t, t_{1}\right)=\varphi(t)$

COROLlaRY 4'. The multiplicity $\left(\chi, \bar{\phi}_{T}^{B}\right)=1$.

Proof. From Lemmas 3 and $4,(\chi, \bar{\varphi})_{T}=\left(\varphi_{T(\mu)}^{T}, \bar{\varphi}\right)=1$. The corollary now follows by Frobenius reciprocity.

Proof of The Theorem. Let $\chi, \varphi$ be as in Proposition 1. From Corollary 2' we have $\mathbf{Q}(\chi)=\mathbf{Q}(\varphi)$. The theorem is therefore proved if $\chi$ can be realized over $Q(\varphi)$. But (with $\bar{\varphi}$ as in Lemma 4) $\bar{\varphi}_{T}^{B}$ is a representation of $B$ in $\mathbf{Q}(\bar{\varphi})=\mathbf{Q}(\varphi)$, which contains a representation whose character is $\chi$ with multiplicity one. Hence $\chi$ can be realized over $Q(\varphi)$.

COROllary. Let the integers $n_{i}$ be as in the proof of Proposition 2, and let $n=$ g.c.d. $\left\{n_{i}\right\}$. Then $\mathbf{Q}\left(\left(q^{n}-1\right) \sqrt{1}\right)$ is a splitting field for the p-regular characters of $B$. 


\section{REFERENCES}

1. G. I. Lehrer, Adjoint groups, regular unipotent elements and discrete series characters, Trans. Amer. Math. Soc. 214 (1975), 249-260.

2. $\longrightarrow$ On a conjecture of Alperin and McKay, Math. Scand. 43 (1978), 5-10.

3. Z. Ohmori, On the Schur indices of reductive groups, Quart. J. Math. Oxford Ser. (2) 28 (1977), 357-361.

Departmant of Pure Mathematics, University of Sydney, Sydney, N.S.W. 2006, Austrania

Current address: Mathematics Institute, University of Warwick, Coventry CV4 7AL, England 